\title{
Zum 100. Geburtstag von Katherine Johnson
}

\author{
Kristina Vaillant
}

\begin{abstract}
Ihre Geschichte wurde mit dem Buch Hidden Figures und dem gleichnamigen Kinofilm einer breiten Öffentlichkeit bekannt: Katherine Johnson, eine afroamerikanische NASA-Mathematikerin, die in den fünfziger und sechziger Jahren maßgeblich dazu beitrug, dass die USA, nach Juri Gagarins erster Erdumrundung, im Wettlauf der Supermächte in der Raumfahrt mit der Sowjetunion zumindest gleichziehen konnte. Am 26. August 2018 feierte die Pionierin ihren 100. Geburtstag.
\end{abstract}

\begin{abstract}
Als Katherine Johnson im Sommer 1953 beim Langley Research Center, der US-amerikanischen Raumfahrtbehörde in Hampton im Bundesstaat Virginia, anfängt, ist sie eine von mehreren Hundert ",computers". „Computer", das war die Berufsbezeichnung für die meist weiblichen Mathematikerinnen, die in drei Schichten an sechs Tagen in der Woche rund um die Uhr mit ihren mechanischen Rechenmaschinen für die Aerodynamik-Experimente der Ingenieure im Windkanal die Berechnungen lieferten. Weil während des Zweiten Weltkrieges viele Männer in den Krieg gezogen waren und es an Arbeitskräften mangelte, warb die Forschungseinrichtung für die Luftfahrt seit 1943 gezielt Frauen und insbesondere auch afroamerikanische Mathematikerinnen an. Separiert von der weißen "computers“-Gruppe, in der einzelne Frauen bereits seit den dreißiger Jahren tätig waren, arbeiteten die Afroamerikanerinnen in einem Neubau, der auf dem westlichen Teil des Geländes lag. Sie waren deshalb auch als die „West Computers“ bekannt.
\end{abstract}

Wir bekamen riesige Datenbögen mit vielleicht 15 oder 20 Spalten und 25 Zeilen. Du hast deine Variable eingesetzt und musstest dann die Gleichungen durchrechnen, das dauerte mehrere Tage,

so beschrieb Johnson die mühselige Arbeit in einem Interview, das sie vor über zehn Jahren dem National Visionary Leadership Project gab, eine Art Oral-History-Plattform, wo ältere Afroamerikaner als Vorbilder ihre Erfahrungen an die jüngere Generation weitergeben.

Für die Ingenieure, die beim National Advisory Committee for Aeronautics (NACA), der Vorgängerorganisation der NASA, die wissenschaftlichen Grundlagen für die Luftfahrt erarbeiteten, waren Johnson und ihre Kolleginnen, ganz gleich welcher Hautfarbe, namenlose Hilfskräfte. Sie nahmen den Ingenieuren die zeitraubenden Berechnungen ab, damit sie ihre wissenschaftlichen Experimente durchführen und Berichte darüber veröffentlichen konnten. Katherine Johnsons sollte 1960 in Langley den ersten Forschungsbericht abliefern, unter dem der Name einer schwarzen Frau stand.

„When computers wore skirts" (Als die Computer noch Kleider trugen), heißt es oft über die Zeit, als wissenschaftliche Berechnungen nicht per Computer, sondern noch per Hand von Assistenten ausgeführt wurden. Dabei war diese Tätigkeit bis zum Zweiten Welt- krieg fast ausschließlich Männersache gewesen. Der USWissenschaftsforscher David Allen Grier schreibt in seinem Buch When Computers were human, es sei ein Beruf für diejenigen gewesen, die zwar in Mathematik gut ausgebildet waren, aber weder über das notwendige Geld, noch über die gesellschaftlichen Verbindungen verfügten, um Karriere zu machen. Neben Afroamerikanern arbeiteten daher, so Grier, auch Juden und irische Einwanderer, Körperbehinderte und generell Menschen aus finanziell prekären Verhältnissen typischerweise als Rechenassistenten. Eine wissenschaftliche Karriere blieb den meisten versagt. Frauen stellten erst mit dem Anbruch der vierziger Jahre die größte Gruppe unter den "computers“.

In einer Schlüsselszene des Films Hidden Figures, der 2017 in Deutschland in die Kinos kam und die Geschichte von drei Frauen aus der West-Computing-Group auf Basis des gleichnamigen Buchs von Margot Lee Shatterly erzählt, steht Katherine Johnson vor der großen Tafel im Büro der Forschungsabteilung Space Task Group. Dorthin war sie versetzt worden, weil die Ingenieure dringend Mathematiker mit dem Spezialgebiet Vektorgeometrie brauchten. Das Langley Research Center war 1958, fünf Jahre nach Johnsons Eintritt in die Behörde, Teil des ersten Programms der NASA für die bemannte Raumfahrt geworden. Damit hofften die USA nach dem SputnikSchock den Wettlauf um den technologischen Vorsprung der Supermächte zu gewinnen. Die Sowjetunion hatte schon im Oktober 1957 mit Sputnik 1 den ersten Satelliten in die Erdumlaufbahn geschossen und damit die Raumfahrt begründet. Die Space Task Group, in die Johnson aufgestiegen war, hatte den Auftrag, die Flugbahnen für die bemannten Missionen ins Weltall zu berechnen: vom Moment des Abschusses am Boden über die Umrundung der Erde in der Umlaufbahn bis zur Landung der Raumkapsel im Atlantik.

Im Film steigt Johnson im Großraumbüro auf die Leiter, notiert ihre Berechnungen auf der übermannshohen Tafel und erläutert, umringt von ihren staunenden Kollegen, warum die bisher eingesetzte Redstone-Rakete nicht geeignet sein würde, eine Raumkapsel bis in die Erdumlaufbahn zu katapultieren. Den NASA-Ingenieuren sei das zu dem Zeitpunkt längst klar gewesen, schreibt Michael Neufeld vom National Air and Space Museum in Washington. Aber auch wenn sich die Szene in Wirklichkeit nie so zugetragen hat - im Film ist sie der symbolische Moment, 


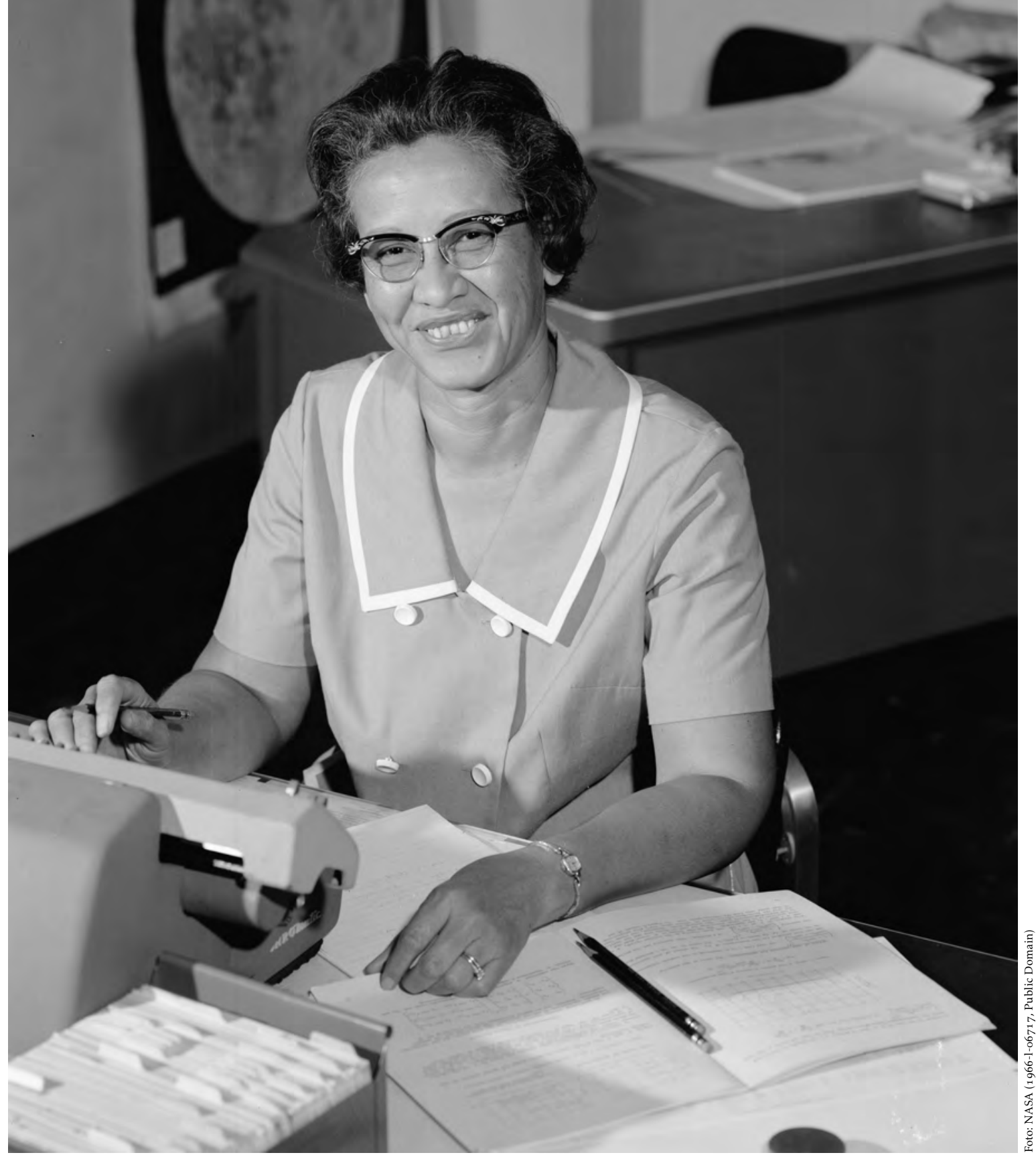

Katherine Johnson im Jahr 1966

in dem Katherine Johnson aus dem Schatten der namenlosen „computers" heraustritt: Abteilungsleiter Al Harrison, ein fiktiver Charakter, gespielt von Kevin Costner, erkundigt sich nach Johnsons ,Auftritt' zum ersten Mal nach dem Namen seiner einzigen schwarzen Mitarbeiterin. Damit ist die Karriere der brillanten Mathematikerin nicht mehr aufzuhalten.

Auch wenn sie gemeinsam mit ihren Kolleginnen aus dem Westflügel nach wie vor in dem Teil des Speiseraums ihre mitgebrachte Mahlzeit essen muss, der mit „,colored only" gekennzeichnet ist, bekommt sie 1959 einen der verantwortungsvollsten Aufträge überhaupt: Die Berechnung der Flugbahn für die erste bemannte Raumkapsel der NASA. Die Mission - ein ballistischer Weltraumflug, keine Erdumrundung - ist erfolgreich: Astronaut Alan Shepard wird am 5. Mai 1961 in den Weltraum katapultiert, erreicht eine Höhe von $187 \mathrm{~km}$ und landet nach
15 Minuten und 22 Sekunden sicher in den Wellen des Atlantiks. Damit war er zwar der erste Amerikaner, aber erst der zweite Mensch im Weltraum. Wenige Wochen zuvor, am 12. April 1961, hatte der sowjetische Kosmonaut Juri Gagarin als erster Mensch die Erde in 106 Minuten einmal umkreist.

Die USA planen nun ebenfalls eine bemannte Raumfahrtmission, die die Erde umkreisen soll. Wieder sind Katherine Johnson und ihre Kollegen mit den Berechnungen für die Flugbahn betraut. Und wieder wird im Film eine dramaturgische Schlüsselszene vor der großen Tafel inszeniert.

Problematisch wird es, wenn die Kapsel von einer elliptischen Umlaufbahn in eine parabolische übergeht, es gibt keine mathematische Formel dafür,

sagt Johnson im Film und wendet sich ihren Kollegen zu. 
Katherine Johnson hatte schon als "computer" unter Beweis gestellt, wie schnell und präzise sie rechnen konnte. Doch worum ging es bei der Berechnung der Flugbahnen? Die größte Herausforderung war, den Astronauten wieder sicher auf die Erde zu bringen. Neben der Berechnung der Umlaufbahn um die Erde war eine zentrale Frage, wann die Bremsraketen vor dem Eintritt in die Erdatmosphäre gezündet werden sollten, damit die Raumkapsel sicher in der vorgesehenen Zone landet.

„Bei den ersten Raumflügen musste man Sicherheitskorridore für den Eintritt in die Erdatmosphäre festlegen“, beschreibt Michael Kirschner, Experte für flugdynamische Berechnung am Deutschen Luft und Raumfahrtzentrum (DLR) in Oberpfaffenhofen das Problem, vor dem die NASA-Mitarbeiter standen.

Wenn die Abbremsung durch die Zündung der Bremsraketen zu stark ist, tritt die Kapsel in einem zu spitzen Winkel in die Erdatmosphäre ein und es besteht die Gefahr, dass die Kapsel verglüht. Bei einem zu schwachen Bremsmanöver, prallt die Kapsel an der Erdatmosphäre ab - und fliegt von der Erde weg. Wie bei einem flachen Stein der in einem flachen Winkel auf die Wasseroberfläche trifft und abprallt.

„Wir können zwar Start und Landung berechnen“, sagt Johnson im Film, „,aber ohne diesen Übergang bleibt die Kapsel im Orbit, wir können sie nicht zurückholen." Mit diesen Worten wendet sie sich wieder den Berechnungen auf der Tafel zu: „Es könnten alte Formeln sein“, spekuliert sie mit Blick auf das Tafelbild, „die das Problem numerisch lösen und nicht analytisch: das Euler-Verfahren."

Das Verfahren, das der Schweizer Mathematiker und Physiker fast zweihundert Jahre zuvor in seinem Buch Institutiones Calculi Integralis beschrieben hatte, wird als Methode der kleinen Schritte bezeichnet. Es ist ein iteratives Verfahren, das häufig bei Problemen angewendet wird, die nicht exakt gelöst werden können, weil es zu viele Variablen gibt. So wie bei der Berechnung der Flugbahn von Objekten wie der Raumkapsel im All. Auf sie wirken verschiedene Kräfte gleichzeitig. Bei den flugdynamischen Berechnungen für den Moment des Eintritts in die Erdatmosphäre muss nicht nur die Rotation der Erde berücksichtigt werden, sondern auch ihr Gravitationsfeld, das je nach Standort variiert, und darüber hinaus die Anziehungskraft des Mondes. Beim Euler-Verfahren wird ein erster Wert berechnet und dieses Ergebnis dann in die nächste Gleichung eingesetzt. Indem man so fortfährt, nähert man sich einer Lösung an. Am Ende dieser Kette von Gleichungen steht zwar kein exaktes Ergebnis, aber die Abweichung davon wird von Gleichung zu Gleichung immer geringer.

Ingenieure kannten die Gleichungen, um das Problem zu beschreiben, aber eben nicht die Verfahren, um sie zu lösen. Dieses mathematische Wissen, das jemand wie Katherine Johnson hatte, das wurde in der Ingenieursausbildung damals sicher nicht vermittelt. Heute gehören die wichtigsten numerischen Lösungsverfahren zum Handwerkszeug,

erklärt Michael Kirschner. Um die Erzählung dramaturgisch zuzuspitzen, wird im Film der Eindruck erweckt, es sei allein Johnsons Verdienst gewesen, das explizite Eulerverfahren als Methode für die numerische Lösung von Differentialgleichungen eingebracht zu haben. Tatsächlich hat mit ihr wohl das gesamte Team an der Kette von Differenzialgleichungen gearbeitet. Das jedenfalls gibt der mathematische Berater der Filmproduktion im Magazin des American Institute of Physics zu Protokoll.

Was 1962 allein in Katherine Johnsons Hand lag, war die Überprüfung der Berechnungen für die Flugbahn der ersten Erdumrundung. Erstmals hatte die NASA dafür einen elektronischen Computer eingesetzt, ein Großrechner vom Typ IBM 7090, der erst kurz zuvor installiert worden war. Bei einer Leistung von 24 ooo Multiplikationen pro Sekunde konnten Rechenoperationen, die per Hand Stunden dauerten, nun innerhalb von Sekundenbruchteilen ausgeführt werden. Doch offenbar gab es bei der NASA Wissenschaftler, die den Rechenkünsten des elektronischen Computers nicht trauten.

Sie baten uns recht häufig, die Berechnungen zu prüfen, bis sie sich ihrer Sache sicher waren. Es war eine Zeit, in der man sich versichern wollte, dass die Computerberechnungen korrekt waren. Wenn sie meint, dass es stimmt, dann stimmt es, so sagten sie,

berichtet Johnson fast vierzig Jahre später in einem Interview.

Die Mission war ein entscheidender Erfolg für die USA. Schließlich galt der technologische Vorsprung, den die Sowjetunion in der Raumfahrt bewiesen hatte, in Zeiten des Kalten Krieges als Bedrohung der nationalen Sicherheit. Der Astronaut John Glenn wurde am 20. Februar 1962 in seiner Raumkapsel Friendship 7 von Cape Canaveral in Florida mit der Atlas-Rakete in den Weltraum geschossen. Innerhalb der nächsten 4 Stunden und 56 Minuten umrundete er die Erde mit einer Geschwindigkeit von 17 ooo Meilen pro Stunde, bevor er 8 oo Meilen südöstlich der Inselgruppe Bermuda sicher im Atlantik geborgen wurde. Den Start verfolgte die ganze Nation voller Spannung vor dem Fernseher und John Glenn wurde nach seiner Rückkehr als Nationalheld gefeiert. Glenn, Johnson und alle anderen, die den ersten bemannten Orbitalflug der NASA vorbereitet hatten, hatten den Beweis erbracht, dass die USA im Wettlauf um die Raumfahrt mit der Sowjetunion mithalten konnten. Die erfolgreiche Raumfahrtmission machte auch Katherine Johnson bekannt. Zumindest in der Afroamerikanischen Presse wurde über ihren Anteil daran berichtet.

Für die NASA war der Eintritt von Johnson und ihren Kolleginnen in die Dienste der amerikanischen Raumfahrtbehörde ein historischer Glücksfall. Die jungen Frauen brachten Talent mit und dazu eine gehörige Portion Mut. Zum Trio im Film Hidden Figures gehören neben 


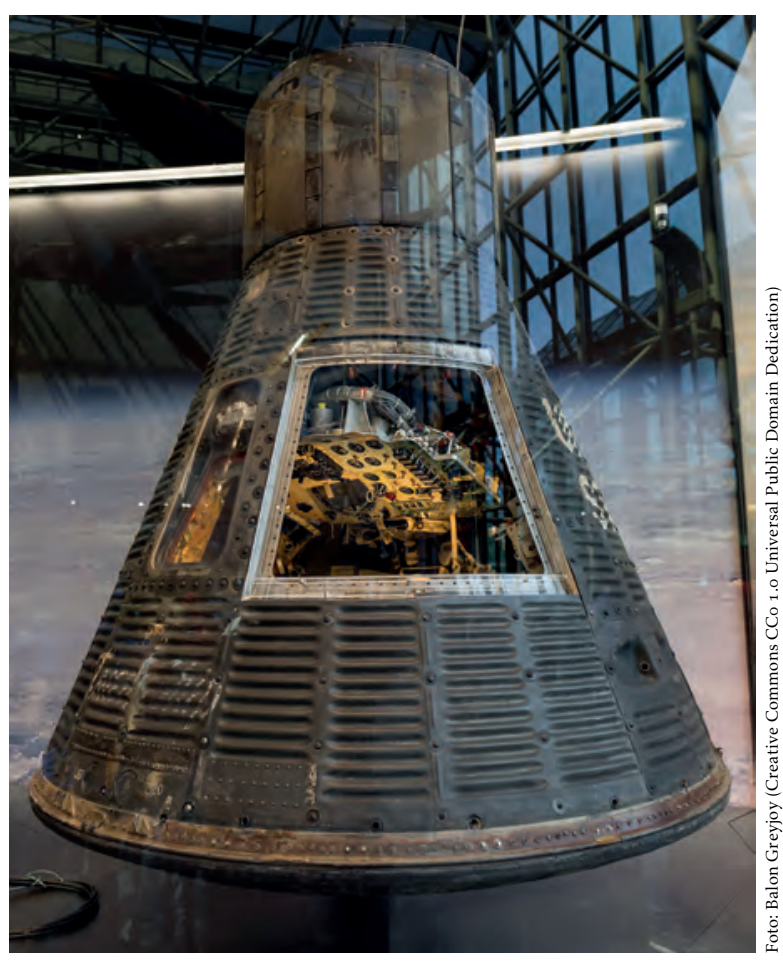

Friendship 7 im Smithsonian Natural Air and Space Museum

Katherine Johnson, von Taraji P. Henson als ein entschlossener und in seiner Ernsthaftigkeit manchmal strenger Charakter gespielt, die im Gegensatz dazu eher kokette Kollegin Mary Jackson, die später die erste schwarze NASA-Ingenieurin werden sollte, und die Leiterin der „West Computers Group“, Dorothy Vaughan. Als die Abteilung 1958 aufgelöst wird, hat sich Letztere längst zur Expertin für die Programmiersprache „Fortran“ fortgebildet und darüber hinaus dafür gesorgt, dass ihre „computers" den Übergang ins Computerzeitalter überstehen.

Doch wie war es möglich, dass diese Frauen in den vierziger und fünfziger Jahren ihre Talente und Persönlichkeiten entfalten konnten? In einer Zeit, in der Afroamerikaner in den Südstaaten der USA durch die JimCrow-Gesetze vom öffentlichen Leben praktisch ausgeschlossen wurden: im Bus durften sie nur in den hinteren Reihen sitzen, die meisten Schulen und Universitäten waren Weißen vorbehalten, es war ihnen untersagt, Mitglied in Vereinen zu werden und in der Bibliothek durften sie, wenn sie überhaupt einen Ausweis bekamen, nur die wenigen Bücher in den für die Schwarzen vorgesehen Regalen ausleihen. In der von weißen Männern dominierten Ingenieurswelt der Luftfahrt mussten sie doppelt Barrieren überwinden - als Frauen und als Schwarze in einer segregierten Gesellschaft.

Katherine Johnson, die als begabte Schülerin Stipendien erhalten und mehrere Klassen übersprungen hatte, war erst 18 Jahre alt, als sie 1937 das West Virginia State College, das ursprünglich ausschließlich für afroamerikanische Studenten gegründet worden war, mit einem Abschluss in Mathematik und Französisch verließ.

$\mathrm{Zu}$ dieser Zeit, Ende der dreißiger Jahre, stand ihr in ihrem Heimatstaat West Virginia im Süden der USA allen- falls eine Stelle als Lehrerin offen, an einer der Schulen, die ausschließlich für Kinder von Afroamerikanern eingerichtet worden waren, weil sie die der Weißen nicht besuchen durften. Auch Johnsons Mutter war Lehrerin gewesen, ihr Vater selbständiger Landwirt und Handwerker. Damit gehörte ihre Familie zur kleinen Mittelschicht, die sich unter den Nachfahren der Sklaven im Süden der USA entwickelt hatte. Die Bildung ihrer vier Kinder lag den Eltern am Herzen. „Mein Vater wollte, dass ich etwas Besseres werde als Grundschullehrerin“, erzählt Katherine Johnson im Interview. Wer als Afroamerikaner nicht zur gut ausgebildeten Mittelschicht gehörte, pflegte die Gärten der weißen Oberschicht, kümmerte sich als Nanny um deren Kinder, war Zugschaffner oder Hausmeister, putzte die Büros im Langley Research Center oder bediente in der Cafeteria.

In der Schule und auch am College wurde Katherine Johnson von Lehrerinnen und Lehrern unterrichtet, die sie inspirierten und förderten. Sie bewunderte ihre Mathelehrerin in der Grundschule und auch in der Highschool war es wiederum eine Lehrerin, die sie überzeugte, Mathematik zu studieren und nicht, wie es ursprünglich ihr Plan war, ausschließlich Sprachen. Auch am College erkannte man ihre Begabung und förderte sie. Der Mathematik-Professor versicherte ihr, sie habe das Zeug als Mathematikerin in der Forschung zu arbeiten und trug seinen Teil dazu bei, indem er ihr Einzelunterricht in Höherer Mathematik erteilte.

Aber es brauchte noch mehr als die Eltern, die für ihre Tochter mehr wollten und Lehrer, die der jungen Frau etwas zutrauten. Denn die Hürden auf dem Weg zur NASA-Mathematikerin waren hoch. Die Jahre nach dem Ende der Wirtschaftskrise in den USA, der Great Depression, die die schwarze Bevölkerung als erste und am heftigsten getroffen hatte, war eine Zeit der Hoffnung und des Aufbruchs. Viele Afroamerikaner hatten bei den Präsidentschaftswahlen 1932 mit Franklin D. Roosevelt erstmals einen Kandidaten der demokratischen Partei zum Präsidenten gewählt, ein Präsident, der mit seinem als „New Deal“ bekannt gewordenen politischen Programm einen modernen Wohlfahrtsstaat errichten wollte. Dennoch schützte die fortschrittliche Politik Roosevelts die schwarze Bevölkerung nicht vor „rassistischer Willkür von Vorgesetzten, Unternehmern, Beamten“, wie Norbert Finzsch und seine Co-Autoren in ihrer Geschichte der African Americans From Benin nach Baltimore feststellen. Bis das Oberste Gericht der USA im November 1956 entschied, dass die Rassentrennung in Bussen verfassungswidrig ist und bis zum Civil Rights Act von 1964, die jegliche Diskriminierung aufgrund von Rasse, Hautfarbe, Religion oder Nationalität verbieten sollte, war es noch ein weiter Weg.

Im Rückblick auf die Geschichte trat die junge CollegeAbsolventin aus der schwarzen Mittelschicht in einem historisch günstigen Moment ins Berufsleben. Arbeitskräfte, vor allem qualifizierte, waren nach dem Ende der Great Depression Anfang der vierziger Jahre in den USA Mangelware und die aufblühende Luftfahrtindustrie, und dazu gehörte auch das Forschungszentrum in Langley, 


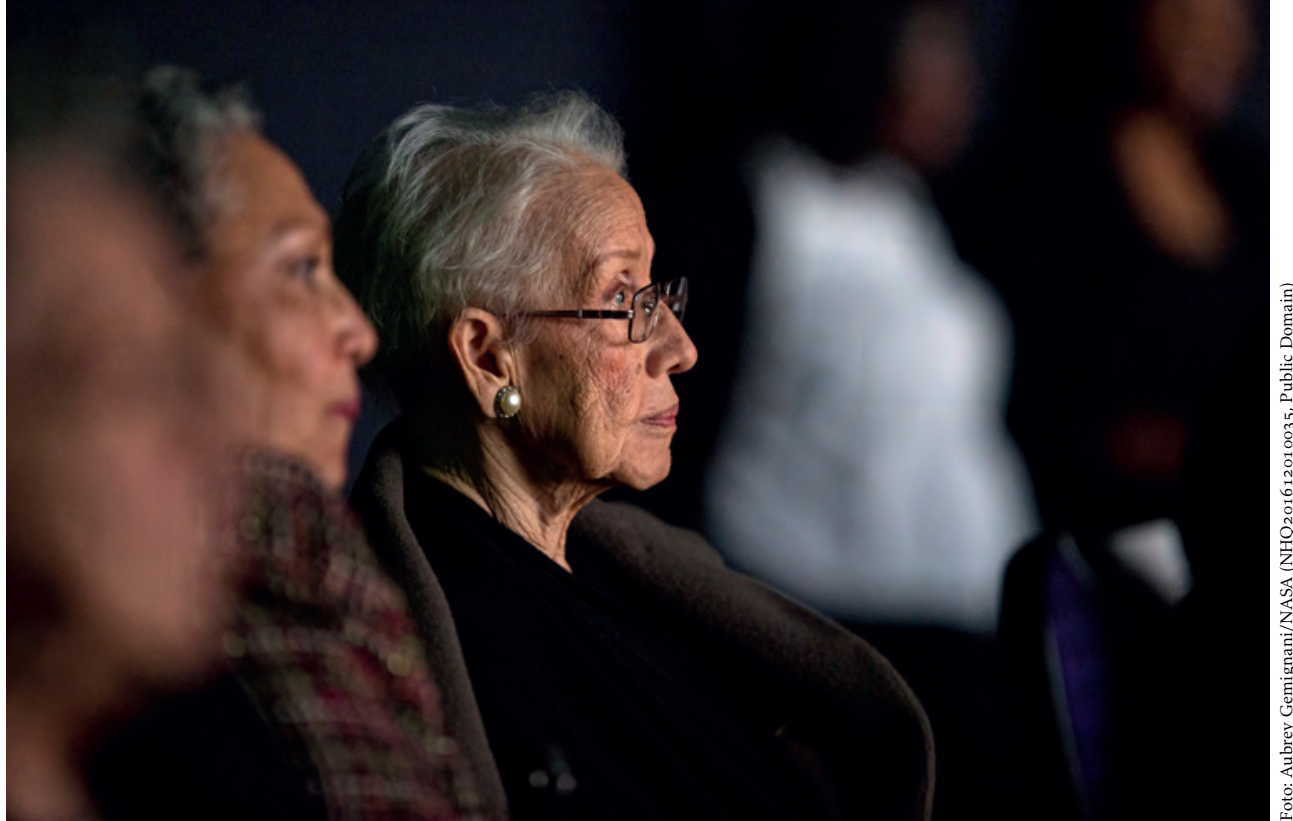

Katherine Johnson am 1.12. 2016 bei der Premiere von Hidden Figures im Virginia Air and Space Center, Hampton, VA

war als kriegswichtig erklärt worden. Die USA rüsteten militärisch auf, Fabriken und Farmen stellten wieder Arbeitskräfte ein. Die Arbeitslosigkeit sank in atemberaubenden Tempo: 1940 zählten die Behörden 7,7 Millionen Arbeitslose, zweieinhalb Jahre später, im Herbst 1942, war die Zahl auf 1,5 Millionen geschrumpft.

Dieser Sog hatte der Bürgerrechtsbewegung in den USA, die unter dem Motto „Jobs for all“ für ökonomische Teilhabe kämpfte, Rückenwind verschafft. Nachdem Asa Philip Randolph, der Gründer und Anführer der ersten schwarzen Gewerkschaft mit einem Marsch nach Washington gedroht hatte, hob Präsident Roosevelt am 25. Juni 1941 mit Erlass 8802 die Rassentrennung in der Rüstungsindustrie auf. Darunter fiel auch das Forschungszentrum der US-amerikanischen Raumfahrtbehörde in Langley. Und weil dort dringend Verstärkung bei den „computers" gebraucht wurde, ließ man in der Personalabteilung die Gelegenheit nicht verstreichen und warb 1943, zwei Jahre nach dem Erlass, in Zeitungsanzeigen erstmals gezielt um Afroamerikanerinnen. Etliche fanden in dieser Zeit auch erstmals Arbeit in Industriebetrieben, das waren Stellen, die nicht nur sicherer, sondern wesentlich besser entlohnt waren als die von Haushälterinnen, Köchinnen oder Wäscherinnen.

Unter Roosevelts Präsidentschaft war Afroamerikanern auch der Zugang zum öffentlichen Dienst erleichtert worden, unter anderem weil fortan Bewerbungen kein Foto mehr beigelegt werden musste. Der Mangel an Arbeitskräften und der Kampf der Bürgerrechtsbewegung Anfang der vierziger Jahre hatte die Barrieren für Afroamerikaner ein Stück abgetragen. Davon profitierten alle: Staat, Wirtschaft und Bevölkerung. Die Emanzipation der Schwarzen bekam einen Schub und das junge Mathetalent aus White Sulphur Springs in West Virginia mit der Einstellung als "computer" in Langley die Chance ihres Lebens.
Katherine Johnson selbst nennt es rückblickend Glück.

Glück ist eine Kombination aus Vorbereitung und Gelegenheit. Wenn Du vorbereitet bist und sich eine günstige Gelegenheit bietet, dann ist es dein Glück, dass Du zur richtigen Zeit am richtigen Ort und vorbereitet auf den Job warst,

mit diesen Worten wird sie auf der Website des National Visionary Leadership Project zitiert. Katherine Johnson arbeitete 33 Jahre, bis zu ihrer Pensionierung 1986, für die NASA. Während sie Karriere machte, zog sie drei Töchter groß, nach dem Tod ihres ersten Mannes zunächst alleine - mit Unterstützung ihrer Mutter. Im Film wird ihr Privatleben eher am Rande miterzählt. Eines wird in diesen Szenen aber deutlich. Ohne Familie, ohne Kirchengemeinde, Freunde und Kollegen, ohne den Rückhalt und die Solidarität der Gemeinschaft wäre das Leben von Johnson anders verlaufen. Dass der Film das herausstellt, anstatt, wie bei anderen Filmen über brillante Mathematiker, die Geschichte eines einsamen Genies zu erzählen, ist auch eine Leistung des Regisseurs Theodore Melfi.

Die Vorlage für den Film lieferte das Buch Hidden Figures von Margot Lee Shatterly, einer Afroamerikanerin und Tochter eines Ingenieurs, der ebenfalls bei der NASA arbeitete. 2016 in den USA erschienen, avancierte das Sachbuch, das ausführlicher als Film über die historischen Umstände berichtet, schnell zum Bestseller. Im Jahr zuvor hatte Präsident Barack Obama die NASA-Mathematikerin mit der Presidential Medal of Freedom, der höchsten Auszeichnung für Zivilisten, gewürdigt. Dass fast sechs Jahrzehnte verstreichen mussten, ehe ihre Leistungen gewürdigt wurden, zeigt, dass der historische Moment, der sie möglich machte, eine Dynamik in Gang setzte, auf die die Gesellschaft noch gar nicht vorbereitet war. Frauen wie Katherine Johnson schon. 